\title{
Research Article Structural Optimization of Slender Robot Arm Based on Sensitivity Analysis
}

\author{
Zhong Luo, ${ }^{1}$ Xueyan Zhao, ${ }^{2}$ Le Liang, $^{1}$ and Fei Wang' \\ ${ }^{1}$ School of Mechanical Engineering \& Automation, Northeastern University, Shenyang 110819, China \\ ${ }^{2}$ Department of Automatic Control and Systems Engineering, University of Sheffield, \\ Mappin Street, Sheffield S1 3JD, UK
}

Correspondence should be addressed to Xueyan Zhao, zhaoxueyan1773@163.com

Received 30 June 2012; Revised 3 December 2012; Accepted 11 December 2012

Academic Editor: Gordon Huang

Copyright @ 2012 Zhong Luo et al. This is an open access article distributed under the Creative Commons Attribution License, which permits unrestricted use, distribution, and reproduction in any medium, provided the original work is properly cited.

\begin{abstract}
An effective structural optimization method based on a sensitivity analysis is proposed to optimize the variable section of a slender robot arm. The structure mechanism and the operating principle of a polishing robot are introduced firstly, and its stiffness model is established. Then, a design of sensitivity analysis method and a sequential linear programming (SLP) strategy are developed. At the beginning of the optimization, the design sensitivity analysis method is applied to select the sensitive design variables which can make the optimized results more efficient and accurate. In addition, it can also be used to determine the scale of moving step which will improve the convergency during the optimization process. The design sensitivities are calculated using the finite difference method. The search for the final optimal structure is performed using the SLP method. Simulation results show that the proposed structure optimization method is effective in enhancing the stiffness of the robot arm regardless of the robot arm suffering either a constant force or variable forces.
\end{abstract}

\section{Introduction}

Structure optimization is concerned with finding the optimal shape of a structure by the iterative process based on the structural response analysis and sensitivity calculation. Sensitivity analysis is used to determine how "sensitive" a model is to changes in the value of the parameters of the model and to changes in the structure of the model. This paper focuses on parameter sensitivity. The parameter sensitivity is to find the most sensitive parameters to the dynamic behavior of the slender robot arm, and such parameters the main analysis object will in the optimization design. This will reduce the difficulty and improve the efficiency. Remarkable progress has been achieved in the structure optimization during the past three decades [1-3]. For new attempts of structure optimization, various 
methods have been developed. Viljoen et al. discussed the application of finite element and genetic methods to design optimization of thin-walled panels considering buckling effects [4]. Kim and Chang proposed fixed grid based shape optimization inspired from topology optimization techniques [5]. Perezzan and Hernández carried out the design sensitivity analysis of the normal stress in a flexural system [6]. Li et al. improved the efficiency for the stress sensitivity calculation beneficially by introducing an integrated virtual load system [7]. Zhao et al. the dynamic characteristics of flexible redundant parallel robot by the sensitivity analysis and the optimization design and represented the displacement responses of the moving platform are investigated through simulation [8,9]. Jarmai et al. published the works on design optimization of welded orthogonally stiffened cylindrical shells in which constraint functions were expressed in explicit forms, and several new mathematical optimization methods were used [10]. Akbari et al. compared the continuum-discrete (C-D) with discrete-discrete (D-D) approaches in detail which presented shape sensitivity formulations for design-dependent loadings [11]. Uhm and Youn eliminated limitations of a shell formulation based on NURBS or T-splines and extended T-spline FEM to the analysis of shells using a mapping scheme [12]. In the field of shape optimal design, Cho and Ha used exact geometric models to enhance shape sensitivities of the isogeometric framework [13].

It should be pointed out that most of the previous studies on sensitivity analysis were carried out based on the discrete approaches, in which the sensitivities of mechanical properties were considered as the design parameters. The sensitivity analysis results can be used to select the optimization variables, but these analysis results ignored the relationship among different mechanical properties. For example, when the structure stiffness increases, it may lead to the increase of mass. Therefore, it is necessary to improve the sensitivity analysis method in the future work to determine the final optimization variables. These variables must be more sensitive to the mechanical properties than the mass to ensure the accuracy of optimization results. Besides, there were very limited theories and studies on the structure optimization of slender robot arm. Nevertheless, the mechanical properties of slender robot arm (such as stiffness, equivalent stress, and inherent frequency, etc) are greatly affected by its own structure features, so further researches are very important, in both analytical and application aspects, toward the structure optimization of slender robot arm.

In this paper, a sensitivity analysis-based optimization program of ANSYS parametric design language (APDL) has been developed to perform structure optimization of a slender robot arm which belongs to a 3-DOF innerwall grinding robot of the solid-propellant rocket engines. Firstly, the main structure mechanism of the grinding robot is presented, and the stiffness model of the robot arm is established. Then, a sensitivity analysis method which has two functions for the structure optimization is proposed. The first function is to select the sensitive design variables by comparing the ratios of the mechanical performance sensitivities to mass sensitivity of the robot arm. The second one is to improve the convergence in the process of the optimization. Finally, the optimizing objective can be achieved using the SLD method.

\section{Structure Mechanism and Stiffness Model of the Robot Arm}

As shown in Figure 1, the position adjusting mechanism of the inner-wall grinding robot consists of the big arm, the forearm, and the wrist whose sections are rectangular thin-walled structure [14]. The lengths of the three parts are $l_{1}, l_{2}$, and $l_{3}$, respectively. The practical setup is shown in Figure 2. 


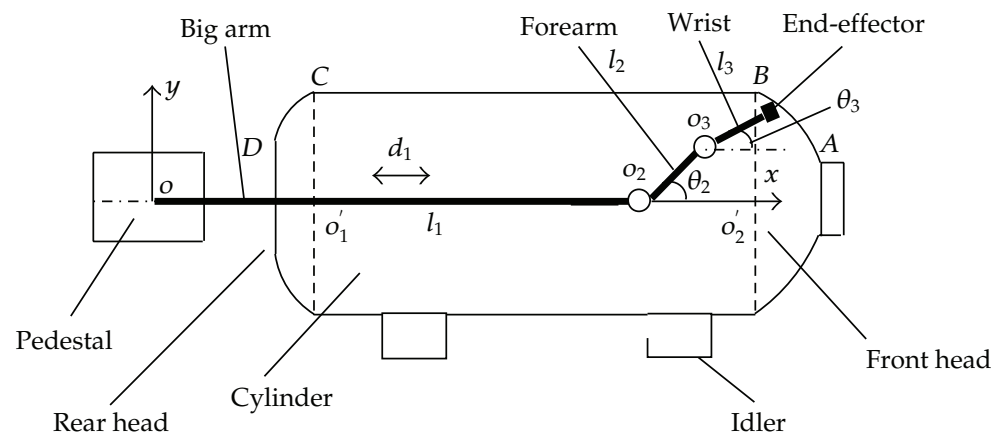

Figure 1: Schematic of the grinding robot.

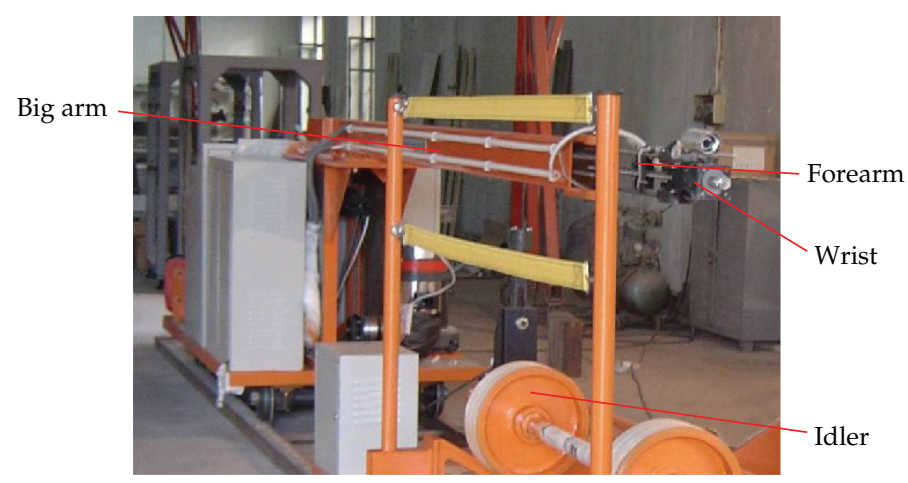

Figure 2: Experimental setup.

The big arm driven by the ball screw can move along the $x$-axis. The forearm can rotate around the joint $\mathrm{O}_{2}$ so that the end-effector can be adjusted to its radial position in order to contact the inner wall of the cylinder. With the rotation of the wrist around the joint $O_{3}$, the angle between the end-effector and the inner wall of the cylinder can be controlled so as to guarantee the end-effector is tangent to the working surface.

In practice, the displacement of the slender robot arm due to its own gravity is much smaller than that induced by the force in the working process. Therefore, the impact of gravity on the displacement is omitted in order to simplify the process of force analysis. When the robot arm is contacted with the working surface, the end-effector will be subjected to two kinds of forces. One is normal pressure $F_{N}$ which is perpendicular to the contact surface. The other is frictional force $F_{f}$ which is tangent to the profile surface of the end-effector. This is shown in Figure 3. In addition, the force $F_{f}^{\prime \prime \prime}$ and the equivalent rotational moment, $T_{f}^{\prime \prime \prime}$ are equivalent forces of $F_{f}$ relative to the point $\mathrm{O}_{3}$.

The force diagram of the robot arm can be obtained after the force condition of the endeffector is applied to each joint. As shown in Figure 4, the normal pressure is converted into one force and one equi-moment acting on each joint. Similarly, the frictional force is converted into one force, one equi-moment, and one rotational equi-moment.

Both big arm and forearm are variable cross-section cantilevers which are reducible to constant section cantilever beam for the approximate calculation. Let the height of section at the end of the big arm as $h_{\xi}$ and $h_{\eta}$, respectively, the simplified height $h_{1}$ is equal 


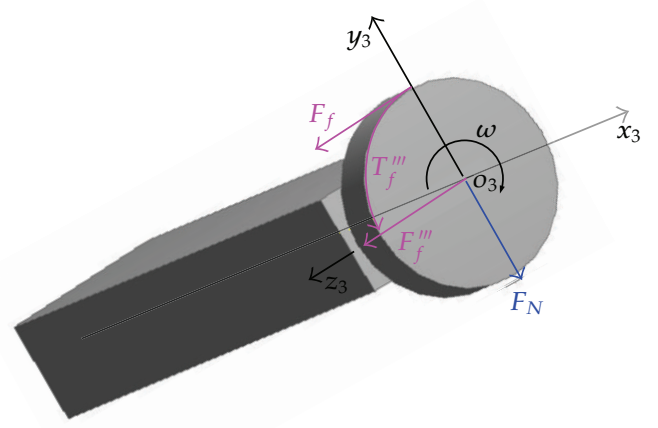

Figure 3: Force condition of the end-effector.

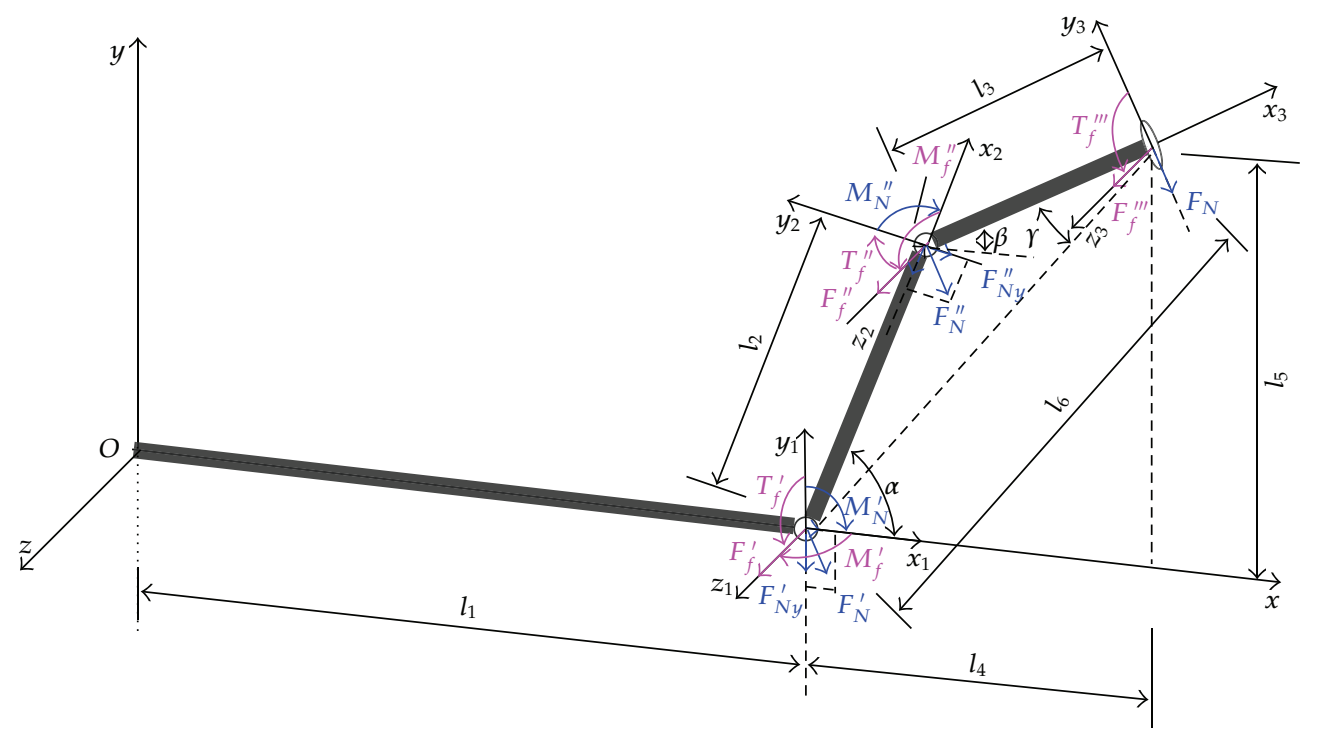

Figure 4: Force diagram of the robot arm.

to $\left(h_{\xi}+h_{\eta}\right) / 2$. The simplified forearm height $h_{2}$ can be calculated by using the same method. Through the topological shape optimization, the relationship between the height and the width of the robot arm can be preliminarily determined as $k_{h}=b_{i} / h_{i}$. For the purpose of guaranteeing the strength and stability of the robot arm, the thickness and height should be satisfied with the relation of $\delta_{i}=k_{\delta, i} h_{i}$. Both elastic and frictional forces work, respectively, in mutual perpendicular plane, so the superposition principle can be used for force analysis. 
The bending displacements for each part of the robot arm which are caused by the normal pressure $F_{N}$ are as follows:

$$
\begin{aligned}
& r_{1}=\frac{F_{N y}^{\prime} l_{1}^{3}}{3 E I_{1}}+\frac{M_{N}^{\prime} l_{1}^{2}}{2 E I_{1}}=\frac{12 l_{1}^{2} F_{N}}{E k_{I, 1} \delta_{1}^{4}}\left(\frac{l_{1} \cos \beta}{3}+\frac{l_{4} \cos \beta+l_{5} \sin \beta}{2}\right), \\
& r_{2}=\frac{F_{N y}^{\prime \prime} l_{2}^{3}}{3 E I_{2}}+\frac{M_{N}^{\prime \prime} l_{2}^{2}}{2 E I_{2}}=\frac{12 l_{2}^{2} F_{N}}{E k_{I, 2} \delta_{2}^{4}}\left[\frac{l_{2} \cos (\alpha-\beta)}{3}+\frac{l_{3}}{2}\right], \\
& r_{3}=\frac{F_{N} l_{3}^{3}}{3 E I_{3}}=\frac{12 l_{3}^{3} F_{N}}{3 E k_{I, 3} \delta_{3}^{4}}
\end{aligned}
$$

The rotation angles of big arm and forearm are

$$
\begin{aligned}
& \theta_{1}=\frac{F_{N y}^{\prime} l_{1}^{2}}{2 E I_{1}}+\frac{M_{N}^{\prime} l_{1}}{E I_{1}}=\frac{12 l_{1} F_{N}}{E k_{I, 1} \delta_{1}^{4}}\left(\frac{l_{1} \cos \beta}{2}+l_{4} \cos \beta+l_{3} \sin \beta\right), \\
& \theta_{2}=\frac{F_{N y}^{\prime \prime} l_{2}^{2}}{2 E I_{2}}+\frac{M_{N}^{\prime \prime} l_{2}}{E I_{2}}=\frac{12 l_{2} F_{N}}{E k_{I, 2} \delta_{2}^{4}}\left[\frac{l_{2} \cos (\alpha-\beta)}{2}+l_{3}\right]
\end{aligned}
$$

where $k_{I, i}=\left(k_{h} / k_{\delta, i}^{4}\right)-\left(\left(k_{h} / k_{\delta, i}\right)-2\right)\left(\left(1 / k_{\delta, i}\right)-2\right)^{3}, l_{4}=l_{2} \cos \alpha+l_{3} \cos \beta, l_{5}=l_{2} \sin \alpha+l_{3} \sin \beta$, $E$ is the elastic modulus of the material, $I_{i}$ is the rotational inertia of the $i$ th section, $k_{I, i}$ is the scale factor of the rotational inertia which relies on $k_{\delta, i}$ and $k_{h}$.

According to (2.1) (2.5), the displacement of the robot arm along the $y$-axis can be deduced as

$$
\begin{aligned}
R_{y} & =r_{1}+\theta_{1} l_{6} \cos (\beta+\gamma)+r_{2} \cos \alpha+\left(\theta_{2} l_{3}+r_{3}\right) \cos \beta \\
& =\frac{12 F_{N}}{E}\left(\frac{K_{1} l_{1}^{3}}{\delta_{1}^{4}}+\frac{K_{2} l_{2}^{3}}{\delta_{2}^{4}}+\frac{l_{3}^{3}}{3 k_{I, 3} \delta_{3}^{4}} \cos \beta\right)
\end{aligned}
$$

where, $l_{6}=\sqrt{l_{2}^{2}+l_{3}^{2}+2 l_{2} l_{3} \cos (\alpha-\beta)}, \gamma=\arcsin \left(l_{2} \sin (\alpha-\beta) / l_{6}\right)$,

$$
\begin{aligned}
& K_{1}=\frac{\cos \beta}{3 k_{I, 1}}+\frac{l_{4} \cos \beta+l_{5} \sin \beta+l_{6} \cos \beta \cos (\beta+\gamma)}{2 k_{I, 1} l_{1}}+\frac{\left(l_{4} l_{6} \cos \beta+l_{3} l_{6} \sin \beta\right) \cos (\beta+\gamma)}{k_{I, 1} l_{1}^{2}}, \\
& K_{2}=\frac{\cos \alpha \cos (\alpha-\beta)}{3 k_{I, 2}}+\frac{l_{3} \cos \alpha+l_{3} \cos \beta \cos (\alpha-\beta)}{2 k_{I, 2} l_{2}}+\frac{l_{3}^{2} \cos \beta}{k_{I, 2} l_{2}^{2}} .
\end{aligned}
$$

The displacement along the $x$-axis is

$$
R_{x}=\theta_{1} l_{6} \sin (\beta+\gamma)+r_{2} \sin \alpha+\left(\theta_{2} l_{3}+r_{3}\right) \sin \beta=\frac{12 F_{N}}{E}\left(\frac{K_{3} l_{1}^{3}}{\delta_{1}^{4}}+\frac{K_{4} l_{2}^{3}}{\delta_{2}^{4}}\right)
$$


where $K_{3}=\left(\left(l_{6} \cos \beta / 2 k_{I, 1} l_{1}\right)+\left(l_{4} l_{6} \cos \beta+l_{3} l_{6} \sin \beta\right) / k_{I, 1} l_{1}^{2}\right) \sin (\beta+\gamma)$,

$$
K_{4}=\frac{\sin \alpha \cos (\alpha-\beta)}{3 k_{I, 2}}+\frac{l_{3} \sin \alpha+l_{3} \sin \beta \cos (\alpha-\beta)}{2 k_{I, 2} l_{2}}+\frac{l_{3}^{2} \sin \beta}{k_{I, 2} l_{2}^{2}} .
$$

The bending displacement $r_{i}^{\prime}$ along the $z$-axis which is produced by the equi-moment $M_{f}$ can be calculated by using the same method shown in (2.1) (2.3). The robot arm can be simplified as the space beam to calculate the twist angle $\varphi_{i}$ which is caused by the rotational equi-moment $T_{f}$. According to the free torsion theory, the twist angle can be expressed as

$$
\varphi_{i}=\frac{T_{f} l_{i}}{G I_{i}^{\prime}}=\frac{T_{f} l_{i} s_{i}}{4 G A_{i}^{2} \delta_{i}}=\frac{T_{f} l_{i}}{2 G k_{I, i}^{\prime} \delta_{i}^{4}}
$$

where $k_{I, i}^{\prime}=\left(\left(\left(k_{h}+1\right) / k_{\delta, i}\right)-2\right) /\left(\left(1 / k_{\delta, i}\right)-1\right)^{2}\left(\left(k_{h} / k_{\delta, i}\right)-1\right)^{2}, G=E / 2(1+\mu)$. Besides, $s_{i}$ is the length of the midline of the section, $A_{i}$ is the size of the area surrounded by the midline, $G$ is the shear modulus of the material, and $\mu$ is the Poisson ratio.

Then the displacement of the robot arm along the $z$ axis is

$$
R_{z}=r_{1}^{\prime}+\varphi_{1} l_{5}+r_{2}^{\prime}+\varphi_{2} l_{3} \sin (\alpha-\beta)+r_{3}^{\prime}=\frac{F_{f}}{E}\left(\frac{K_{5} l_{1}^{3}}{\delta_{1}^{4}}+\frac{K_{6} l_{2}^{3}}{\delta_{2}^{4}}+\frac{4 l_{3}^{3}}{k_{I, 3} \delta_{3}^{4}}\right)
$$

where $K_{5}=4 / k_{I, 1}+6 l_{4} / k_{I, 1} l_{1}+E l_{5}^{2} / 2 G k_{I, 1}^{\prime} l_{1}^{2}, K_{6}=4 / k_{I, 2}+6 l_{3} \cos (\alpha-\beta) / k_{I, 2} l_{2}+E l_{3}^{2} \sin ^{2}(\alpha-$ B) $/ 2 G k_{I, 2}^{\prime} l_{2}^{2}$.

In summary, the bending defection of the robot arm shown in (2.6), (2.8), and (2.11) can be rewritten as follows:

$$
\mathbf{R}=\left[\begin{array}{l}
R_{x} \\
R_{y} \\
R_{z}
\end{array}\right]=\left[\begin{array}{ccc}
\frac{12 K_{3} F_{N}}{E} & \frac{12 K_{4} F_{N}}{E} & 0 \\
\frac{12 K_{1} F_{N}}{E} & \frac{12 K_{2} F_{N}}{E} & \frac{4 \cos \beta F_{N}}{K_{I, 3} E} \\
\frac{K_{5} F_{f}}{E} & \frac{K_{6} F_{f}}{E} & \frac{4 F_{f}}{K_{I, 3} E}
\end{array}\right]\left[\begin{array}{l}
\frac{l_{1}^{3}}{\delta_{1}^{4}} \\
\frac{l_{2}^{3}}{\delta_{2}^{4}} \\
\frac{l_{3}^{3}}{\delta_{3}^{4}}
\end{array}\right] .
$$

Based on the above calculations, the function relationships among the rotation angles of the forearm and the wrist $(\alpha, \beta)$, the wall thicknesses of the robot arms $\left(\delta_{1}, \delta_{2}, \delta_{3}\right)$, and the displacement $(\mathbf{R})$ can be obtained, so that the effect of wall thicknesses on stiffness of the robot arm is identified when the robot arm is in any working state. It can be indicated by (2.12) that even a subtle change of the wall thickness can have a relatively substantial impact on the displacement. Therefore, the bending stiffness of the robot arm can be enhanced significantly through the optimal allocation of the wall thicknesses. 


\section{Design Sensitivity}

Design sensitivity analysis, that is, the calculation of quantitive information on how the response of a structure is affected by changes of the variables that define its shape, plays an important role in structural shape optimization. There are generally two approaches to calculate the sensitivities [15]. The first one is based on differentiation of the finite element equations $[16,17]$, and the second one is the finite difference method. The first approach uses a discretized structural model. For this model, the sensitivities are obtained by differentiating the finite element matrix equations with respect to the design variables. Though this method can achieve a relatively exact solution, the application of precise methods always leads to perfect but impractical results for the complex model. So the finite difference method has been considered as a reference method for linear elastic problems, since it performed well for most of the cases. Of course, it has serious limitations related to truncation and round-off errors. But these errors can usually be minimized by using an appropriate step size of the design perturbation.

In this study, the first-order forward finite difference is used to calculate the design sensitivities of the objective functions and constraint functions.

The design sensitivities for objective functions can be written as

$$
S_{f, i}=\frac{\partial \mathrm{f}}{\partial x_{i}}=\frac{f\left(x_{i}+\Delta x_{i}\right)-f\left(x_{i}\right)}{\Delta x_{i}}
$$

and, for $j$ th constraint function,

$$
S_{g, i}=\frac{\partial g_{j}}{\partial x_{i}}=\frac{g_{j}\left(x_{i}+\Delta x_{i}\right)-g_{j}\left(x_{i}\right)}{\Delta x_{i}}
$$

where $\Delta x_{i}$ is a small perturbation in the variable $x_{i}$.

Though the bigger absolute value of the above sensitivities, the faster response of the corresponding variable is, only sensitivities may not be enough to evaluate the impact of the variables on the objective function or the constraint functions. This is due to the fact that if a variable is sensitive to both mechanical properties and mass, this variable may not necessarily be the key variable to the mechanical properties, because actually optimization may achieve the purpose of controlling the properties at the cost of increasing the mass of the structure. Based on the above consideration, the influence extent of each variable on the optimization objective can be assessed by comparing the ratios of the mechanical performance sensitivities to mass sensitivity of the robot arm. It can be calculated by the following equations:

$$
\begin{aligned}
& \frac{S_{f, i}}{S_{m, i}}=\frac{f\left(x_{i}+\Delta x_{i}\right)-f\left(x_{i}\right)}{m\left(x_{i}+\Delta x_{i}\right)-m\left(x_{i}\right)}, \\
& \frac{S_{g, i}}{S_{m, i}}=\frac{g\left(x_{i}+\Delta x_{i}\right)-g\left(x_{i}\right)}{m\left(x_{i}+\Delta x_{i}\right)-m\left(x_{i}\right)},
\end{aligned}
$$




\section{Optimization Model and Solution Algorithms}

The mathematical model for design optimization problem can be formulated as

$$
\begin{aligned}
& \min \quad f(\mathbf{X}) \\
& \text { s.t. } \quad g_{j}(\mathbf{X}) \leq 0 \quad j=(1,2, \ldots, m), \\
& x_{i L} \leq x_{i} \leq x_{i U} \quad i=(1,2, \ldots, n),
\end{aligned}
$$

where $\mathbf{X}=\left(x_{1}, x_{2}, \ldots, x_{n}\right)$ is the vector of the design variables, $x_{i L}$ and $x_{i U}$ are lower and upper limit of the design variables, $f(\mathbf{X})$ is the objective function, $g_{j}(\mathbf{X})$ is the constraint functions, $m$ is the number of constraints, and $n$ is the number of design variables.

In this study, sequential linear programming (SLP) is used to minimize the objective function with respect to the constraint equations. In the SLP algorithm, the objective and constraints functions are approximated with linear extensions at the current design point during the optimization iteration. Then the original problem is transformed into the following linear programming problems [18]:

$$
\begin{aligned}
& \min \quad f\left(\mathbf{x}_{0}\right)+\nabla^{\mathrm{T}} f\left(\mathbf{x}_{0}\right) \Delta \mathrm{x} \\
& \text { s.t. } \quad g_{j}\left(\mathbf{x}_{0}\right)+\nabla^{\mathrm{T}} g_{j}\left(\mathbf{x}_{0}\right) \Delta \mathrm{x} \leq 0 \\
& x_{i L} \leq x_{i} \leq x_{i U},
\end{aligned}
$$

where the $\nabla^{T} f\left(\mathbf{x}_{0}\right)$ and $\nabla^{T} g_{j}\left(\mathbf{x}_{0}\right)$ are derivative gradients of the objective function and constraint functions, respectively. The linear programming is solved with the Lamke pivot algorithm to update design. These approximations and solution procedures are repeated until the convergence is reached.

For this approach, the optimum solution is always obtained at one of the vertices formed by the design constraints. Since there is the finite number of such vertices in the feasible region, a systematic approach such as the simplex method is used to search for the optimum solution among these vertices.

In a SLP method, the result of each iteration is expected to be a better solution. Since the linear programming technique is used to find the optimum solution for a nonlinear problem, some measures are taken to improve the optimization process. In this study a heuristic iterative algorithm based on the design sensitivity is employed to control the changes in the design variables. At a given design stage, the design variables are updated iteratively as

$$
\mathbf{X}^{(k+1)}=\mathbf{X}^{(k)}-\alpha^{(k)} \mathbf{S}^{(k)}
$$

where, $\mathbf{X}$ denotes the vector of the design variables, $\mathbf{S}$ is the vector of the sensitivities values, and $\alpha$ is the scale moving step along that direction, and the superscript $k$ indicates the optimization cycle number. In most cases, the search direction is chosen along the negative of the vector of the sensitivities, as shown in (4.3), to make the optimization process more effective. 
The success of the optimization process depends on the scale of moving step which can improve the convergence to a large degree. For efficiency and effectiveness of the algorithm, the moving step $\alpha$ is now determined from the following equations:

$$
f(\mathbf{X})^{(k+1)} \approx f(\mathbf{X})^{k}+\left(\mathbf{S}^{(k)}\right)^{T} \cdot \Delta \mathbf{X}^{(k)}=f(\mathbf{X})^{k}-\alpha^{(k)}\left|\mathbf{S}^{(k)}\right|^{2}
$$

Then, it is suggested

$$
\alpha^{(k)}=\frac{\mu f(\mathbf{X})^{k}}{\left|\mathbf{S}^{(k)}\right|^{2}}
$$

where $\left|\mathbf{S}^{(k)}\right|$ indicates the Euclidean norm of the vector of the sensitivities and $\mu$ is the reduction of the objective value to be attained in the present design cycle.

The basic algorithm for the structural shape optimization based on mathematical programming is shown in Figure 5 and can be summarized in the following steps.

Step 1. Establish the objective function $f(\mathbf{X})$ and constraint function $g(\mathbf{X})$ as defined in (4.1).

Step 2. Build the finite element model of the structure.

Step 3. Carry out a finite element analysis using the design variables.

Step 4. Evaluate the sensitivities of the objective and constraint functions of the current design.

Step 5. Calculate the scale moving step with (4.5).

Step 6. Using a suitable optimization algorithm, such as SLP, generate a new structural shape which satisfies the constraints.

Step 7. If the new structural shape is not optimum, update the model to Step 2 with (4.3), otherwise stop.

To realize the structure optimization task, different principles such as structural analysis, automatic mesh generation, finite element analysis, sensitivity analysis, and mathematical programming are interrelated. As shown in Figure 5, the flowchart of the optimization process is provided by a computer program coded, and the ANSYS is used for automatic mesh generation and finite element analysis, but the value of the constraints is obtained using finite differences.

\section{Results}

In order to validate the above analysis and optimization approach, the design of the robot arm of the inner-wall grinding robot of the solid-propellant rocket engines which was introduced at the beginning of the paper was performed.

When the robot arm is at the position shown in Figure $6\left(\alpha=58.5^{\circ}, \beta=27.8^{\circ}\right)$, its working accuracy is at the lowest level in the operation process. Therefore, in this paper, the 


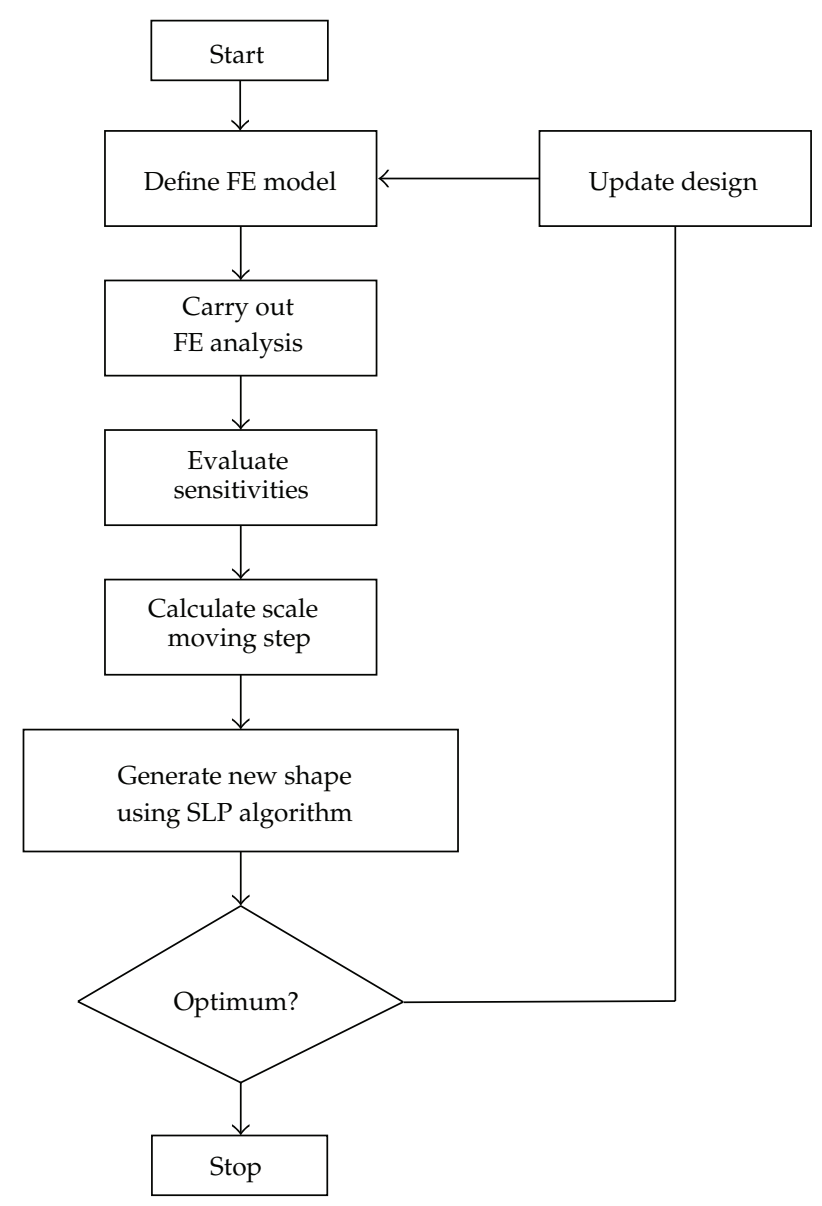

Figure 5: Flowchart of the structural optimization.

structure of the robot arm at this position state is optimized in order to ensure the mechanical properties can still meet the engineering requirements in the whole work condition. As shown in Figure 6, the position of the variables $\left(x_{1}-x_{10}\right)$ has been pointed out. Where $x_{1}, x_{2}$, and $x_{3}$ are design variables of the major arm thickness, $x_{4}, x_{5}$, and $x_{6}$ are design variables of the joint thickness, $x_{7}$ and $x_{8}$ are design variables of the length and width of the big arm, respectively, $x_{9}$ and $x_{10}$ are design variables of the length and width of the forearm, respectively. Due to the special working conditions, the length of the robot arm cannot be changed, so it cannot be considered as design variables.

Due to the bigger the displacement of the robot arm, the smaller will be its stiffness, so the stiffness sensitivity can be replaced by the displacement sensitivity. The sensitivities of some significant mechanical properties are performed for the different design variables; the results are listed in Table 1.

The ratios of the mechanical performance sensitivities to mass sensitivity of the robot arm are demonstrated in Figure 7 which is implemented by in-house MATLAB code. Thus, the major variables which have important impacts on the mechanical properties and relatively insignificant effect on the mass can be found. 


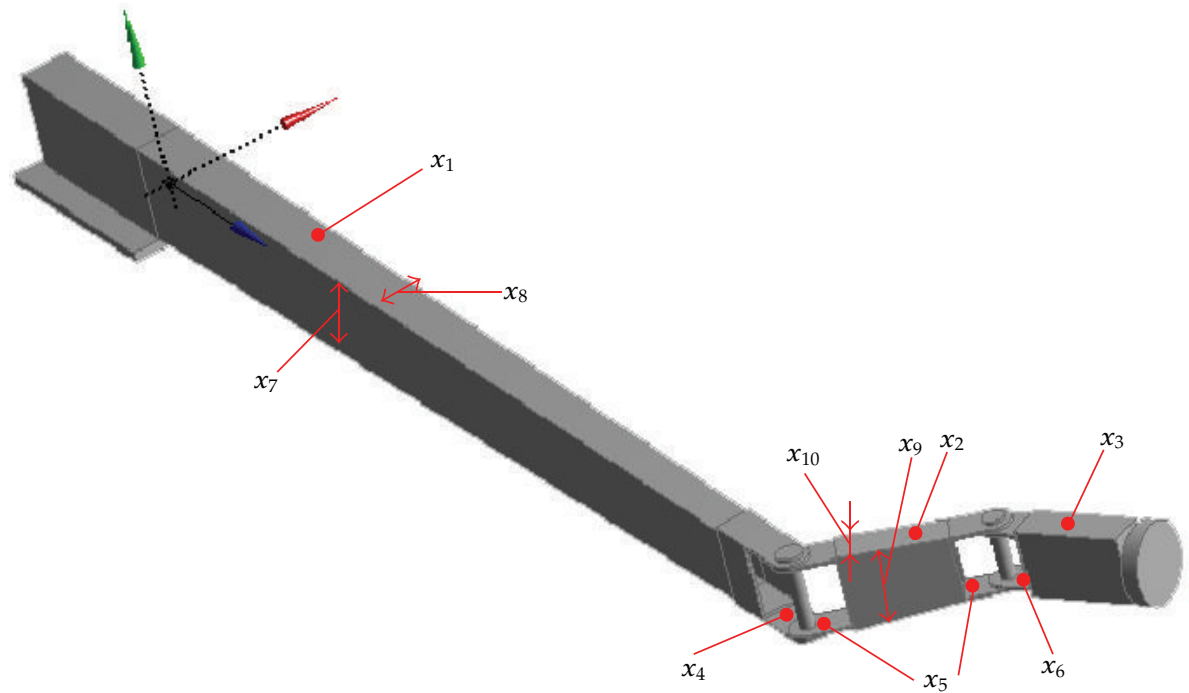

Figure 6: The model of the robot arm and design variables.

Table 1: Variable sensitivity results.

\begin{tabular}{lcccccc}
\hline $\begin{array}{l}\text { Design } \\
\text { variable }\end{array}$ & $\begin{array}{c}\text { Initial } \\
\text { value } \\
(\mathrm{mm})\end{array}$ & $\begin{array}{c}\text { Mass } \\
\text { sensitivity } \\
\left(S_{m}\right)\end{array}$ & $\begin{array}{c}\text { Bending } \\
\text { displacement } \\
\text { sensitivity }\left(S_{d}\right)\end{array}$ & $\begin{array}{c}\text { Equivalent } \\
\text { stress } \\
\text { sensitivity }\left(S_{\sigma}\right)\end{array}$ & $\begin{array}{c}\text { Modal strain } \\
\text { energy } \\
\text { sensitivity }\left(S_{e}\right)\end{array}$ & $\begin{array}{c}\text { Inherent } \\
\text { frequency } \\
\text { sensitivity }\left(S_{f}\right)\end{array}$ \\
\hline$x_{1}$ & 5 & $6.32 e-002$ & $6.99 e-002$ & 0.114 & 0.169 & 0.157 \\
$x_{2}$ & 5 & $8.24 e-002$ & $2.51 e-002$ & $0.17 e-002$ & 0.110 & $5.17 e-002$ \\
$x_{3}$ & 5 & $3.01 e-002$ & 0.226 & 0.127 & 0.153 & $8.38 e-002$ \\
$x_{4}$ & 6 & $5.73 e-002$ & $2.33 e-002$ & $7.29 e-003$ & $3.58 e-002$ & $6.26 e-002$ \\
$x_{5}$ & 6 & 0.127 & $0.21 e-002$ & $0.13 e-002$ & $4.42 e-002$ & $5.51 e-002$ \\
$x_{6}$ & 7 & 0.158 & $1.82 e-002$ & $0.15 e-002$ & $1.33 e-002$ & $3.15 e-002$ \\
$x_{7}$ & 180 & $8.78 e-003$ & $3.02 e-002$ & $5.34 e-002$ & $4.81 e-002$ & $4.17 e-002$ \\
$x_{8}$ & 90 & 0.106 & $3.63 e-002$ & 0.155 & $8.20 e-002$ & $7.64 e-002$ \\
$x_{9}$ & 150 & 0.109 & 0.231 & 0.382 & 0.269 & 0.234 \\
$x_{10}$ & 75 & $5.16 e-004$ & $0.15 e-002$ & $0.16 e-002$ & $0.18 e-002$ & $0.15 e-002$ \\
\hline
\end{tabular}

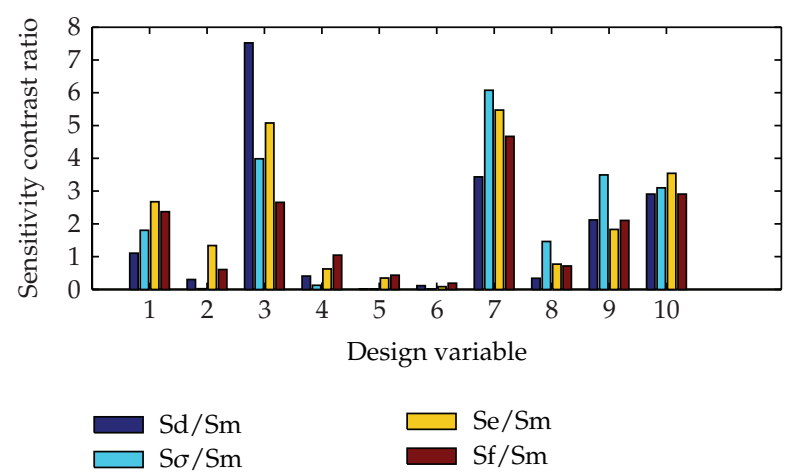

Figure 7: Contrast of sensitivities. 
Table 2: Initial properties value.

\begin{tabular}{lccccc}
\hline Properties & Mass $(\mathrm{kg})$ & $\begin{array}{c}\text { Bending } \\
\text { displacement }(\mathrm{mm})\end{array}$ & $\begin{array}{c}\text { Equivalent } \\
\text { stress }(\mathrm{Mpa})\end{array}$ & $\begin{array}{c}\text { Modal strain } \\
\text { energy }(\mathrm{mJ})\end{array}$ & $\begin{array}{c}\text { Natural } \\
\text { frequency }(\mathrm{Hz})\end{array}$ \\
\hline Value & 74.21 & 1.31 & 20.00 & 44.28 & 130.47 \\
\hline
\end{tabular}

Figure 7 indicates obviously that the sensitivity ratios of variables 2, 4, 5, 6, and 8 are much less than the remaining ones. If the relevant variables are optimized to improve the mechanical properties, the mass of the robot arm will increase significantly. As a result, and it is unsuitable to optimize these variables. Therefore, variables $1,3,7,9$, and 10 are selected as the subsequent optimization design variables. Definitely, the sensitivity analysis can greatly reduce the number of design variables, so it can not only shorten distinctly the optimization time, but also make the optimization results easier to comply with the design requirements.

Before the robot arm is optimized, the value of its properties is calculated by finite element analysis. The results are listed in Table 2.

The constraint conditions are set as the following four parts. (1) The mass of the robot arm must be controlled within certain range. (2) The maximum equivalent stress meets the structure strength requirement. (3) The modal strain energy cannot exceed the limit value. (4) In order to reduce the vibration, the natural frequency of the robot arm should be away from the working frequency

$$
\begin{gathered}
\frac{\left(m_{b}-m_{a}\right)}{m_{b}} \geq \Delta_{m} \\
\frac{\sigma_{s}}{\sigma_{\max }} \geq S_{a} \\
U \leq U_{\varepsilon} \\
f_{w}<\eta f_{d} \text { or } f_{w}>\eta f_{d}, \quad \eta>1.2,
\end{gathered}
$$

where $m_{b}$ and $m_{a}$ are the mass of the robot arm before and after optimization, $\Delta_{m}$ is the variation range of the mass. $\sigma_{s}$ and $\sigma_{\max }$ are yield strength and maximum equivalent stress, respectively, and $S_{a}$ is the safety factor. $U$ is the modal strain energy, $U_{\varepsilon}$ is the limit value. $f_{w}$ is the working frequency, $f_{d}$ is the natural frequency and $\eta$ is the limit coefficient of the frequency, range.

The objective function is taken as the bending deflection. In this paper, it is replaced by the displacement $\mathbf{R}$.

Figure 8 demonstrates the evolutionary history of the bending displacement of the robot arm which decreases from $1.31 \mathrm{~mm}$ to $0.67 \mathrm{~mm}$, that is, by about $49.01 \%$. It can be seen clearly from Figure 8 that the bending displacement of the robot becomes stable after iteration 29. Therefore, the optimization program is convergent well through the iterative computing. In addition, the optimal properties of robot arm are listed in Table 3.

It can be indicated from Tables 2 and 3 that the mass of robot arm is reduced by $9.1 \%$, namely, from $74.211 \mathrm{~kg}$ to $67.464 \mathrm{~kg}$. So the robot arm becomes lighter after optimization. Besides, bending displacement, equivalent stress, modal strain energy, and natural frequency are all decreased. Thus, the mechanical properties of the robot arm are greatly improved.

The bending displacement and equivalent stress of the robot arm in the optimum state are shown in Figures 9 and 10, respectively. 
Table 3: Optimization design results.

\begin{tabular}{lcccccc}
\hline $\begin{array}{l}\text { Design } \\
\text { variables }\end{array}$ & $\begin{array}{c}\text { Optimal } \\
\text { value (mm) }\end{array}$ & Mass (kg) & $\begin{array}{c}\text { Optimization results } \\
\text { displacement }(\mathrm{mm})\end{array}$ & $\begin{array}{c}\text { Equivalent } \\
\text { stress (MPa) }\end{array}$ & $\begin{array}{c}\text { Modal strain } \\
\text { energy (mJ) }\end{array}$ & $\begin{array}{c}\text { Natural } \\
\text { frequency (Hz) }\end{array}$ \\
\hline$x_{1}$ & 5.1842 & & & & & \\
$x_{3}$ & 4.7505 & & & & & \\
$x_{7}$ & 180.35 & 67.46 & 0.67 & 15.40 & 42.74 & 120.63 \\
$x_{9}$ & 146.06 & & & & & \\
$x_{10}$ & 78.562 & & & & & \\
\hline
\end{tabular}

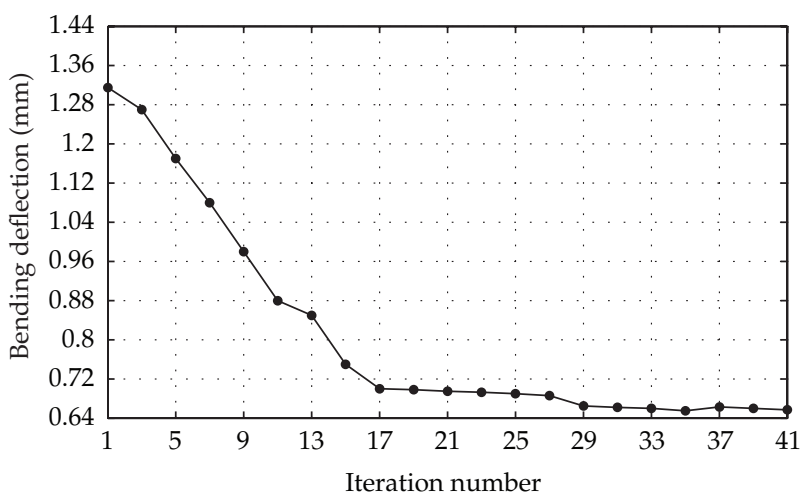

Figure 8: Evolutionary history of the bending deflection in the design process.

The above optimization results are obtained when the robot arm is suffering static force. However, in the practical working process, the load is changed with time due to the heterogeneity of the processing material, the vibration caused by the electromotor, and so on. The mechanical testing on the inner-wall grinding robot in practical working process indicates that the robot arm is subjected to a sinusoidal loading. When the robot arm is remodeled according to the optimal variables, its displacement with the above variable load is obtained by ANSYS and shown in Figure 11.

It can be demonstrated by Figure 11 that the displacement of the robot arm is significantly declined. Therefore, the optimization method is also effective to the case when the robot arm is subjected to a variable load.

\section{Conclusions}

By taking a 3-DOF inner-wall grinding robot of the solid-propellant rocket engines as an example, a structure optimization programme has been developed for a slender robot arm based on the finite element method and the sensitivity analysis strategy in this paper. The search for the optimal structure is performed using the SLP technique. The following conclusions are drawn.

(1) The sensitivity analysis method and a sequential linear programming (SLP) strategy are applicated and developed for the structure optimization of slender arm 
Type: total deformation

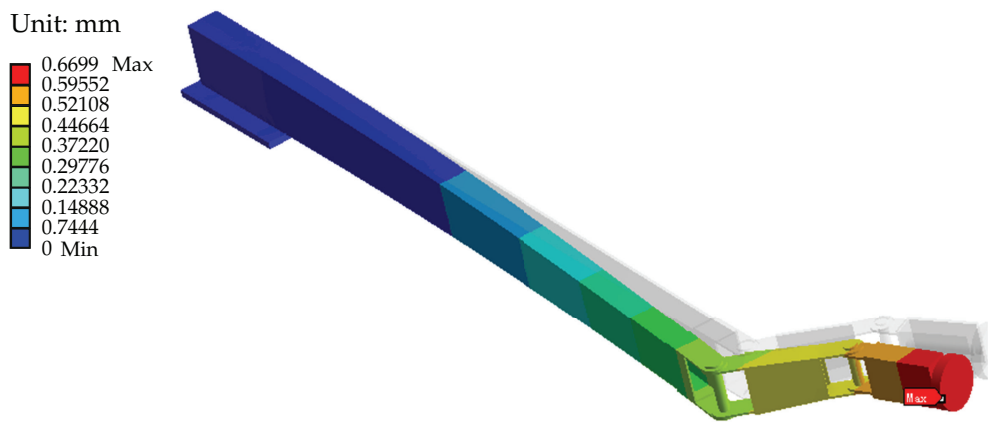

Figure 9: Bending displacement of the robot arm.

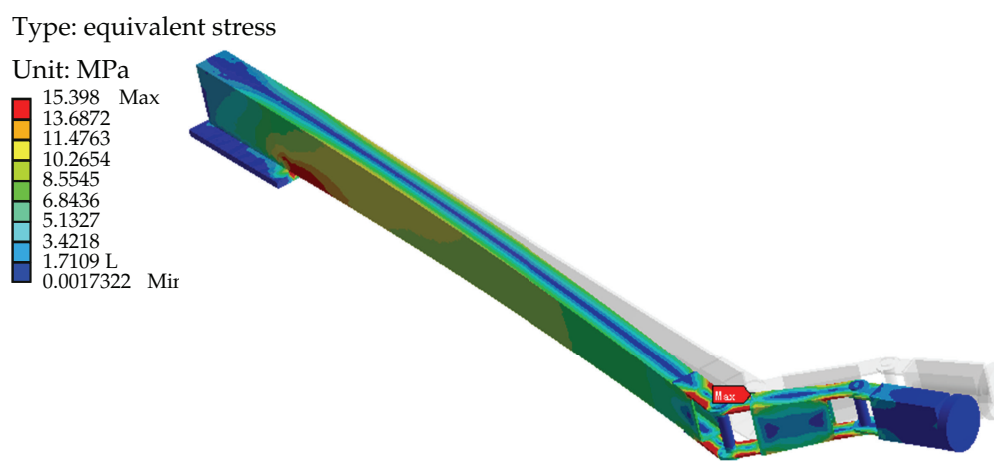

Figure 10: Equivalent stress of the robot arm.

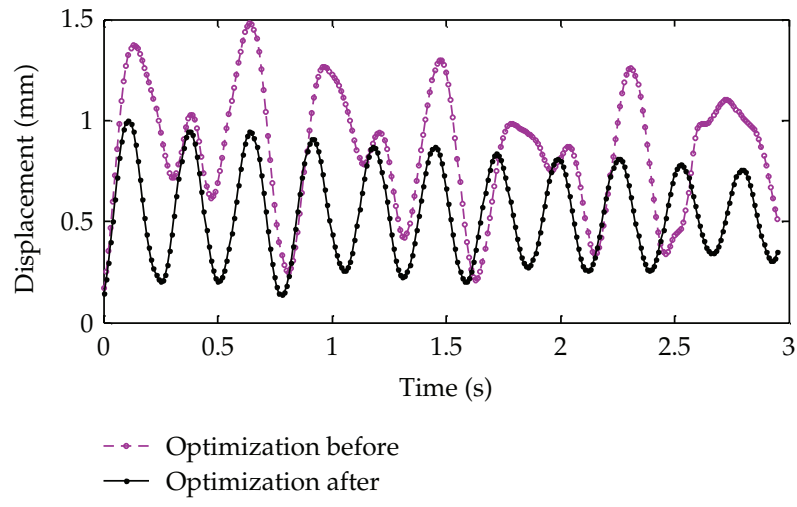

Figure 11: Displacement response curves of the robot arm.

robot. The main sensitive design variables are selected by comparing the ratios of the mechanical performance sensitivities to mass sensitivity of the robot arm.

(2) The sensitivity analysis method can not only be used to reduce the number of the design variables before optimizing for the purpose of increasing efficiency and 
accuracy, it can also be used to determine the scale of moving step which will improve the convergency during the optimization process.

(3) The displacement of the robot arm with optimal structure is reduced significantly no matter it is suffering constant force or variable force.

(4) This study is application oriented and can be a useful example of structural design optimization for engineers.

\section{Nomenclature}

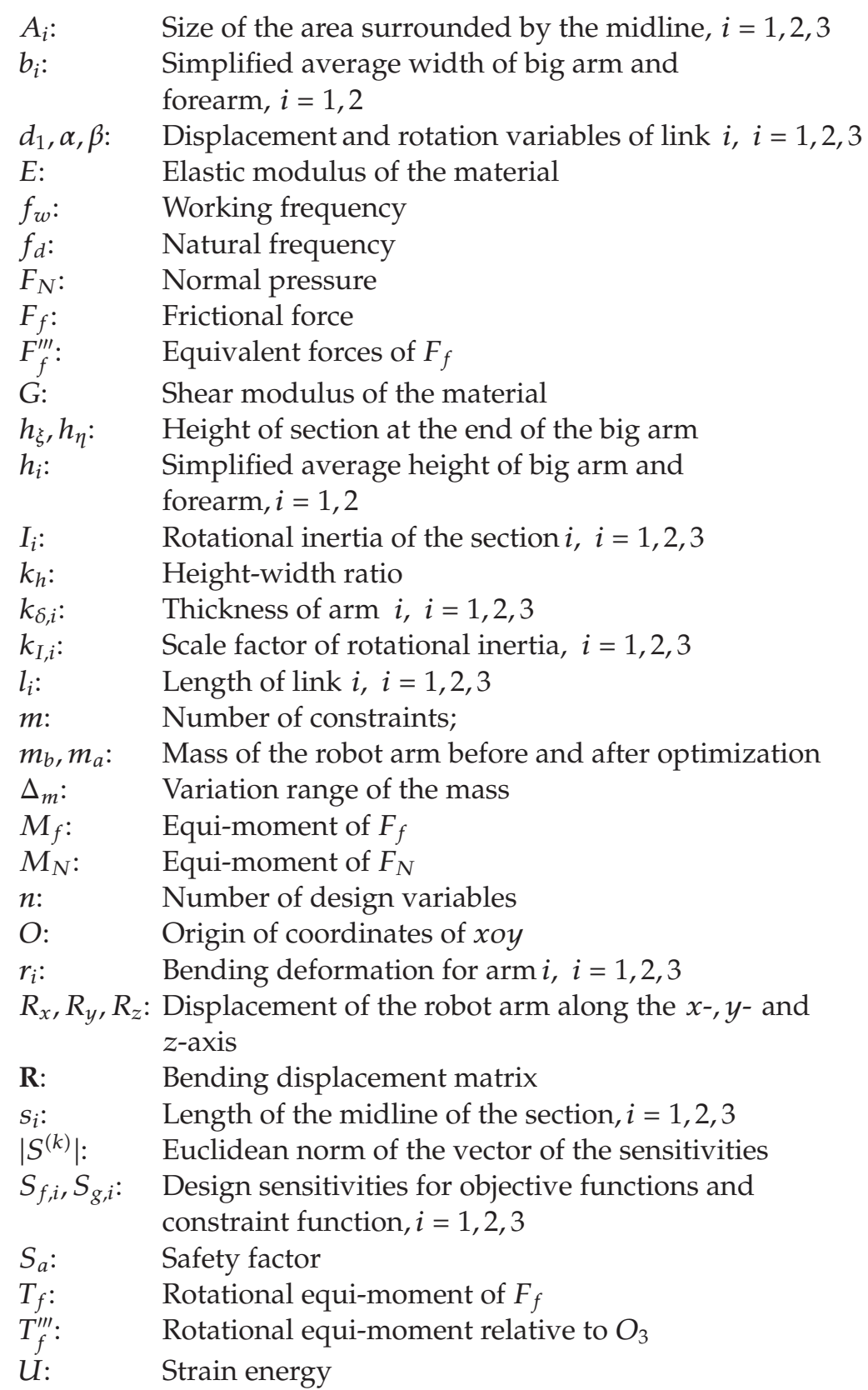




$\begin{array}{ll}U_{\varepsilon}: & \text { Limit value } \\ \Delta x_{i}: & \text { A small perturbation in the variable } x_{i}, i=1,2,3 \\ \mathbf{X}=\left(x_{1}, x_{2}, \ldots, x_{n}\right): & \text { Vector of the design variables; } \\ x_{i L}, x_{i U}: & \text { Lower and upper limit of the design variables, } i=1,2,3 \\ f(\mathbf{X}): & \text { Objective function } \\ g_{j}(\mathbf{X}): & \text { Constraint functions, } i=1,2,3 \\ \nabla^{T} f\left(\mathbf{x}_{0}\right), \nabla^{T} g_{j}\left(\mathbf{x}_{0}\right): & \text { Derivative gradients of the objective function and } \\ & \text { constraint functions } \\ \sigma_{s}, \sigma_{\max }: & \text { Yield strength and maximum equivalent stress; } \\ \varphi_{i}: & \text { The twist angle, } i=1,2,3 \\ \mu: & \text { Poisson's ratio } \\ \theta_{i}: & \text { Rotational angles of big arm and forearm, } i=1,2 \\ \delta_{i}: & \text { Wall thicknesses of arm } i, i=1,2,3 \\ \eta: & \text { Limit coefficient of the frequency range. }\end{array}$

\section{Acknowledgments}

This work is supported by the National Science Foundation of China (51105064), the National Program on Key Basic Research Project (2012CB026000), and the Fundamental Research Funds for the Central Universities of China (N100403007).

\section{References}

[1] G. N. Vanderplaats, Numerial Optimization Techniques for Engineering Design: With Application, McGraw-Hill, New York, NY, USA, 1984.

[2] R. T. Haftka and Z. Gurdal, Elements of Structural Optimization, Kluwer Academic Publishers, Boston, Mass, USA, 1992.

[3] M. Pourazady and Z. Fu, "An integrated approach to structural shape optimization," Computers and Structures, vol. 60, no. 2, pp. 279-289, 1996.

[4] A. Viljoen, A. G. Visser, and A. A. Groenwold, "Computationally efficient analysis and optimization of stiffened thin-walled panels in shear," Journal of Aircraft, vol. 42, no. 3, pp. 743-747, 2005.

[5] N. H. Kim and Y. Chang, "Eulerian shape design sensitivity analysis and optimization with a fixed grid," Computer Methods in Applied Mechanics and Engineering, vol. 194, no. 30-33, pp. 3291-3314, 2005.

[6] J. C. Perezzan and S. Hernández, "Analytical expressions of sensitivities for shape variables in linear bending systems," Advances in Engineering Software, vol. 34, no. 5, pp. 271-278, 2003.

[7] Q. Li, G. P. Steven, and Y. M. Xie, "Evolutionary structural optimization for stress minimization problems by discrete thickness design," Computers and Structures, vol. 78, no. 6, pp. 769-780, 2000.

[8] Y. Zhao, F. Gao, X. Dong, and X. Zhao, "Dynamics analysis and characteristics of the 8-PSS flexible redundant parallel manipulator," Robotics and Computer-Integrated Manufacturing, vol. 27, no. 5, pp. 918-928, 2011.

[9] Y. J. Zhao, "Dimensional synthesis of a three translational degrees of freedom parallel robot while considering kinematic anisotropic property," Robotics and Computer-Integrated Manufacturing, vol. 29, no. 1, pp. 169-179, 2013.

[10] K. Jarmai, J. A. Snyman, and J. Farkas, "Minimum cost design of a welded orthogonally stiffened cylindrical shell," Computers and Structures, vol. 84, no. 12, pp. 787-797, 2006.

[11] J. Akbari, N. H. Kim, and M. T. Ahmadi, "Shape sensitivity analysis with design-dependent loadings-equivalence between continuum and discrete derivatives," Structural and Multidisciplinary Optimization, vol. 40, no. 1-6, pp. 353-364, 2010.

[12] T. K. Uhm and S. K. Youn, "T-spline finite element method for the analysis of shell structures," International Journal for Numerical Methods in Engineering, vol. 80, no. 4, pp. 507-536, 2009.

[13] S. Cho and S. H. Ha, "Isogeometric shape design optimization: exact geometry and enhanced sensitivity," Structural and Multidisciplinary Optimization, vol. 38, no. 1, pp. 53-70, 2009. 
[14] Z. Luo, S. Yang, Y. Sun, and H. Liu, "Optimized control for dynamical performance of the polishing robot in unstructured environment," Shock and Vibration, vol. 18, no. 1-2, pp. 355-364, 2011.

[15] H. Uysal, R. Gul, and U. Uzman, "Optimum shape design of shell structures," Engineering Structures, vol. 29 , no. 1, pp. 80-87, 2007.

[16] P. Pedersen and C. L. Laursen, "Design for minimum stress concentration by finite elements and linear programming," Journal of structural mechanics, vol. 10, no. 4, pp. 375-391, 1982.

[17] D. Wang, W. H. Zhang, and J. S. Jiang, "Truss shape optimization with multiple displacement constraints," Computer Methods in Applied Mechanics and Engineering, vol. 191, no. 33, pp. 3597-3612, 2002.

[18] B. Chen, G. Liu, J. Kang, and Y. Li, "Design optimization of stiffened storage tank for spacecraft," Structural and Multidisciplinary Optimization, vol. 36, no. 1, pp. 83-92, 2008. 


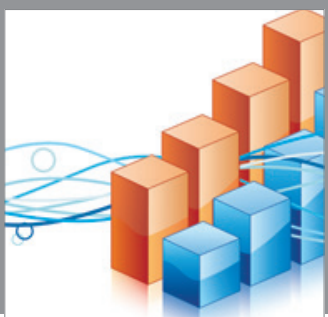

Advances in

Operations Research

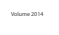

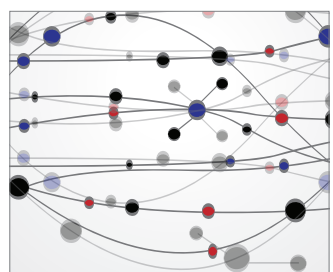

\section{The Scientific} World Journal
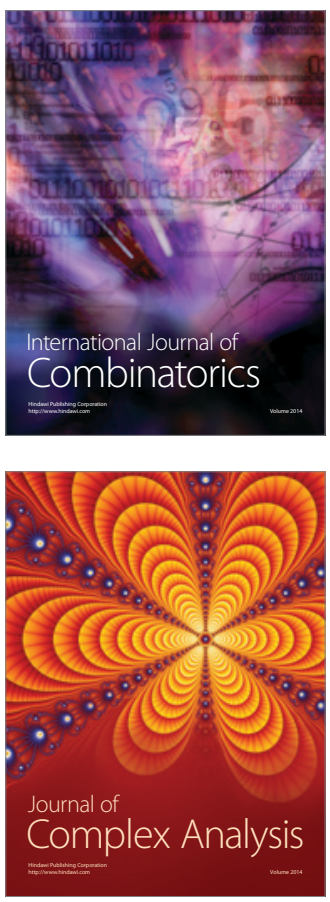

International Journal of

Mathematics and

Mathematical

Sciences
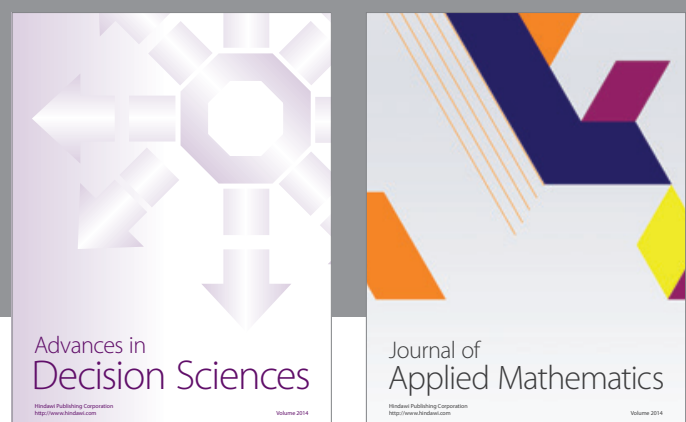

Journal of

Applied Mathematics
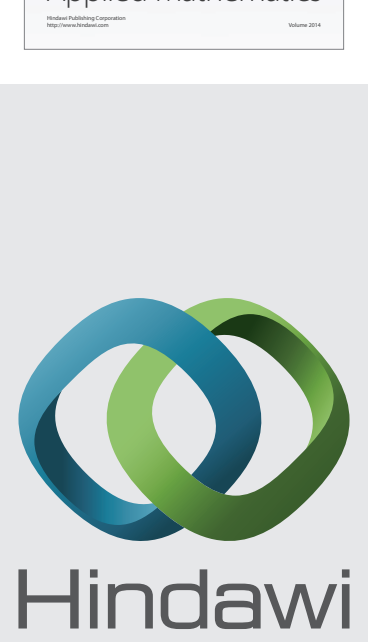

Submit your manuscripts at http://www.hindawi.com
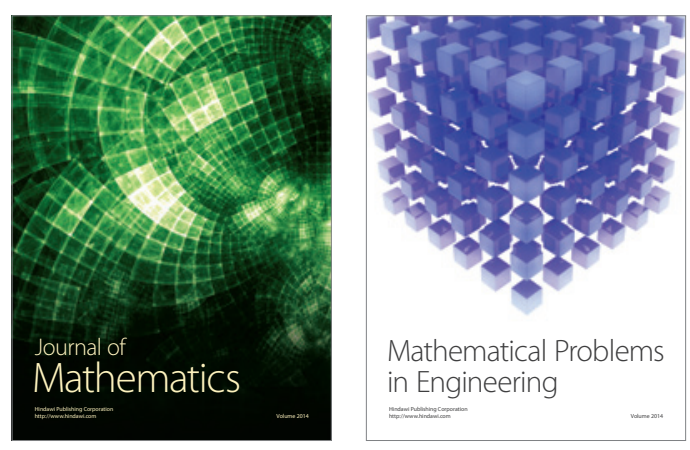

Mathematical Problems in Engineering
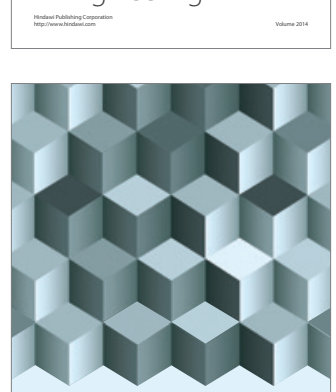

Journal of

Function Spaces
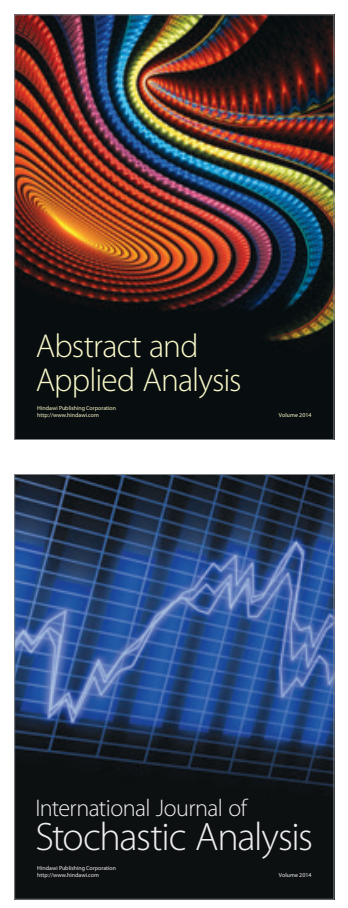

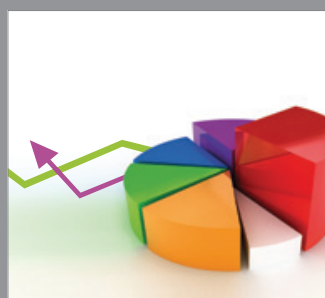

ournal of

Probability and Statistics

Promensencen
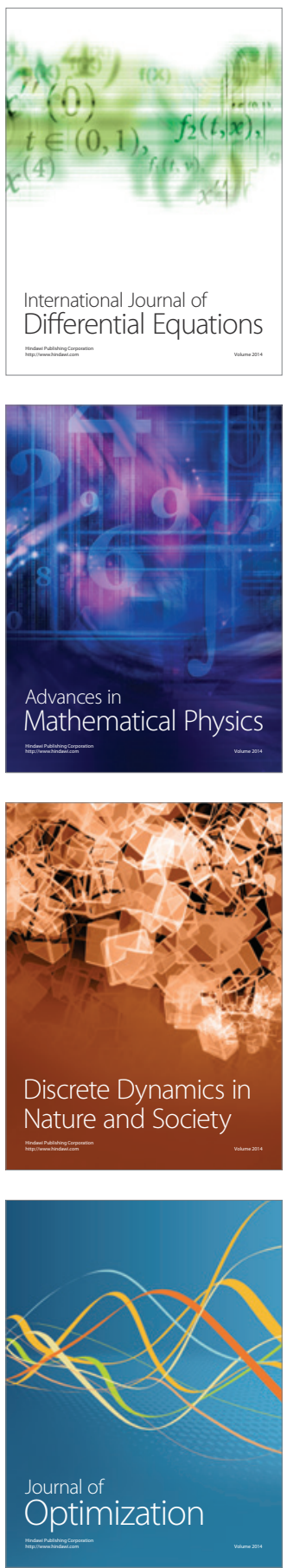